Purpose The objective of this work was to evaluate the features of consultations made by patients in these situations.

Materials and Methods observational prospective study performed in all outpatients who demanded an interview with the pharmacist from 01/03/12 to 31/05/12. Data collected: sex, age, pathology, type of question, resolution (yes/no), and whether the patient was sent to another health professional or not.

Results 48 patients were included $(56.25 \%$ male; mean age 47.25 years). Pathology: 29 HIV; 4 hepatitis C; 3 multiple sclerosis; 3 hepatitis B, and 9 others (one each): lung cancer, renal impairment, rheumatoid arthritis, multiple myeloma, myosarcoma, growth disorder, pulmonary hypertension, glaucoma, and aspergillus infection. Consultations were classified into 9 types showing in brackets the number of each: 1-Drug-drug interactions (14); 2-Apply for extra medication (9); 3-Side effects (8); 4-Dosage and administering(6); 5-Missed or wrong doses(6); 6-Prescription renewal(2); 7-Drug storage(1); 8-Faulty drug(1) and 9-Misunderstanding medical prescription(1). Forty-three consultations were solved by the pharmacist $(89.58 \%)$. In the other 5 cases, patients were sent to the physician: two were taking the treatment incorrectly and needed a special cheque, two needed to renew the prescription and one was suffering severe side effects.

Conclusions The most common consultations were related to pharmacology except for $18,75 \%$ of patients who applied for extra medication (often not possible because of the hospital policy). The pharmacist was able to solve almost $90 \%$ of consultations, sending the patients to their doctors just in cases where their health was compromised or new prescriptions were needed.

No conflict of interest.

\section{DGI-005 ANALYSIS OF LEVOFLOXACIN USE IN GERIATRIC UNITS AT A UNIVERSITY HOSPITAL}

doi:10.1136/ejhpharm-2013-000276.271

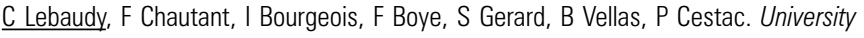
Hospital, Geriatric Department, Toulouse, France

Background Overuse of antibiotics, such as fluoroquinolones and third-generation cephalosporins, is a major cause of the emergence of extended-spectrum beta lactamase producing enterobacteriaceae. The use of levofloxacin in elderly inpatients is widespread.

Purpose We investigated the conditions in which this drug was prescribed.

Materials and Methods From 1st January to 31st March 2012, information was recorded on every new levofloxacin prescription from the geriatric units: indication, dose, duration, patient's medical history, renal function and previous antibiotic. In parallel, levofloxacin consumption was assessed and expressed in terms of the number of Defined Daily Doses (DDD) per 1000 patient-days (PD). The consumption was compared with the data from the French antibiotic network "RAISIN".

Results 87 patients had a levofloxacin prescription: $55 \%$ for community-acquired pneumonia, $20 \%$ for nursing-associated pneumonia, $16 \%$ for nosocomial pneumonia, and $9 \%$ for others indications. $77 \%$ of the patients had previously received another antibiotic (47 amoxicillin/clavulanic acid, 20 ceftriaxone). Among patients without signs of gravity (tachycardia, tachypnea, hypotension), 1 in every 2 received levofloxacin associated with ceftriaxone, although this combination is only for intensive care patients according to the French Society of Infectious Diseases. The mean duration of treatment was 10 days. In 1 in every 2 cases, dosage was too high according to the renal function. As a result, the exposure to levofloxacin was 49 DDD per 1000 PD in acute-care units, and 37 DDD per 1000 PD in skilled units. These results are 4 to 7 times higher than those recorded in the "RAISIN" network. For $20 \%$ of the patients, levofloxacin was ineffective and another line of antibiotic was prescribed.
Conclusions Our results suggest that to reduce exposure to fluoroquinolones we should avoid systematic association with ceftriaxone, prescribe levofloxacin as the second line after amoxicillin/clavulanic acid and reduce dose and duration.

No conflict of interest.

\section{DGI-006 ANALYSIS OF SAVINGS IF THE TREATMENT OF COMMUNITY-ACQUIRED PNEUMONIA (CAP) IS SWITCHED}

doi:10.1136/ejhpharm-2013-000276.272

${ }^{1}$ E Dogliani, ${ }^{1} \mathrm{~F}$ Perrino, ${ }^{2} \mathrm{FG}$ De Rosa, ${ }^{3} \mathrm{~F}$ Cattel, ${ }^{4} \mathrm{G}$ Raineri, ${ }^{5} \mathrm{M}$ Abrate, ${ }^{6} \mathrm{M}$ Paire. ${ }^{1}$ Scuola di Specializzazione in Farmacia Ospedaliera, Università degli Studi di Torino, Cuneo, Italy; ${ }^{2}$ Ospedale Amedeo di Savoia, S.C. Infettivologia, Torino, Italy; ${ }^{3} \mathrm{AOU}$ San Giovanni Battista - Molinette, S.C.Farmacia, Torino, Italy; ${ }^{4}$ ASO S.Croce e Carle, S.C. Malattie Infettive e Tropicali, Cuneo, Italy; ${ }^{5}$ ASO S.Croce e Carle, S.C.Farmacia, Cuneo, Italy; ${ }^{6}$ ASL CN1, S.C Farmacia Territoriale, Cuneo, Italy

Background Levofloxacin exhibits excellent bioavailability as well as pharmacokinetic equivalence between the oral and the parenteral form and is one of the medicines most used in the treatment of CAP. Purpose The purpose of this study is to evaluate the savings that may be achieved by treating patients affected with CAP with sequential treatment (switching from intravenous to oral treatment).

Materials and Methods Both the cost and duration of treatment with levofloxacin were considered. The cost was given by: unitary cost of levofloxacin, cost of the nursing staff, cost of the material for parenteral infusion, cost of the hospitalisation. The duration was considered to be 5 days for patients without complications, 20 days for patients with complications and 10 days as the average in common clinical practise. This model was applied to reality in the S.C. Pneumologia of the ASO S. Croce and Carle of Cuneo. The patients hospitalised for CAP and treated with levofloxacin were individualised through the A.S.400 computerised applications.

Results In 2011351 patients were hospitalised and treated with levofloxacin tablets and/or vials in the Pneumology ward; $90 \%$ of them were suffering from CAP.

For 10 days of treatment the sequential treatment would enable savings equal to $85 € /$ patient. This saving would allow us to treat 12 more patients in a switched treatment regime. For 20 days of treatment the difference would be equal to $205 €$ /patient quantifiable as 14 more patients with CAP treated in hospital without affecting the budget.

Conclusions Oral treatment, as it is equally effective, turns out to be the best therapeutic alternative in terms of savings. In future we will analyse the discharge letters of these patients under the model used in this study, thus assessing the real savings.

No conflict of interest.

\section{DGI-007 ANALYSIS OF THE PRESCRIPTIONS OF ANTIBIOTICS AS LAST RESORT}

doi:10.1136/ejhpharm-2013-000276.273

A Charuel, J Salles, P Guyot, M Legrand-Thapthimdoem. Centre Hospitalier de Mayotte, Mayotte, Mamoudzou, France

Background The composite index on proper use of antibiotics (ICATB) includes surveillance of the ATBs used, evaluation of ATB prescriptions and the existence of an ATB list associated with checking dispensing with limited duration.

Purpose To examine the conformity of ATBs as last resort prescriptions and to promote their proper use.

Materials and Methods 1988 prescriptions emanating from 7 units were investigated between 2009 and 2011, by taking into account 7 criteria: re-evaluation of the need to continue the treatment, conformity with administrative (AR), clinical/biological (CR), pharmaceutical (PR) requirements, the relevance of the 